\title{
Direct linking of autonomic activity with typed text: The CARMEN machine
}

\author{
JAMES W. PENNEBAKER and CARMEN UHLMANN \\ Southern Methodist University, Dallas, Texas
}

\begin{abstract}
A technique that allows researchers to simultaneously record typed text and link each typed word with up to four channels of autonomic nervous system activity is described. The computerbased system is useful for studying the biological correlates of language production. The system, which works with J\&J biofeedback equipment, can be adapted to virtually any monitoring equipment that can provide A/D conversion. Suggestions for data analyses are included.
\end{abstract}

Traditionally, psychophysiologists and researchers interested in autonomic nervous system activity have adopted paradigms in which experimental participants are passive. That is, subjects typically view pictures, hear sounds, receive shocks, or process other types of information presented by the experimenter. The researcher then monitors the subjects' biological responses in ways that are presumably free from movement artifacts. Despite the intuitive appeal of this research strategy, it does not afford an accurate glimpse of many true day-to-day psychological processes in which the individuals are actively thinking and generating ideas. The goal of our research is to have experimental subjects freely type their thoughts and feelings about various topics while psychophysiological activity is continuously measured. Such a strategy allows the researcher to see how the content of the thoughts generated by the subjects is related to autonomic activity.

In order to examine the links between specific words, phrases, and idea units with their physiological correlates, we have developed a relatively simple PC-based system that can be attached to standard biofeedback equipment. The apparatus and computer program needed for this system can be assembled at a reasonable cost with a modest knowledge of electronics and computer programming. We currently refer to the composite system as the Computerized Autonomic Retrieval for Morphemes and Even Neologisms (i.e., CARMEN) machine.

\section{Physiological Monitoring Apparatus}

The basic autonomic measurement system is available from J\&J Enterprises (22797 Holgar Ct. N.E., Poulsbo, WA 98370). Any physiological measuring apparatus, however, can be adapted if one has access to a good computer programmer. The J\&J equipment is commonly used by biofeedback experts in private practice. Consequently,

This research was made possible by National Science Foundation Grant BNS90-21518. Address correspondence to J. W. Pennebaker, Department of Psychology, Southern Methodist University, Dallas, TX 75275 (e-mail: h7dr0001@vm.cis.smu.edu). the system includes far more "bells and whistles" than most researchers want or need.

Physiological hardware. For the measurement of skin conductance level (SCL) at two locations and heart rate (HR), we use the basic I-330 interface (which includes A/D capability), the MH-330 module housing, a P-401 plethysmograph (PPG) module, and the T-68 temperature/ GSR module (including two IG-3 pre-amps) with the 1611 stand-alone adapter. This system with the J\&J USEC computer program costs about $\$ 3,400$.

For a savings of over $\$ 1,000$, one could purchase J\&J's T-601 temperature/GSR module in place of their older T-68. Unfortunately, the newer T-601 system uses direct current for SCL, which is a major problem if the researcher wishes to measure SCL at more than one site at a time. The older T-68 system works on a low-voltage AC system that avoids interference of SCL measurements from site to site. In other words, if you plan to measure SCL at only one location, use the cheaper T-601 solution.

Physiological sensors. For the measurement of SCL, we have been satisfied with $\mathrm{J} \& \mathrm{~J}$ 's $\mathrm{Ag} / \mathrm{AgCl}$ finger electrodes attached to Velcro strips. When used on fingers, the electrodes are placed on the third phalange of subjects' first and third fingers of both hands (Pennebaker, Hughes, \& O'Heeron, 1987). When measuring SCL from the feet, we monitor midway between the ankle and the ball of the foot, with the electrodes approximately $2 \mathrm{~cm}$ apart (Edelberg, 1967). Note that when monitoring at two or more sites and/or using the T-60l equipment, it is absolutely necessary to mark the electrodes so that the same leads go to the same measuring locations for each subject.

Measurement of heart rate using the J\&J P-401 equipment is more tricky. The photoplethysmographs that are supplied by J\&J to be used on the fingers are overly sensitive to subtle changes in ambient lighting and to minuscule body movements. To overcome these problems, we designed and built our own photoplethysmograph sensors that can be attached to the subject's earlobe. A small, inexpensive low-voltage yellow light-emitting-diode (LED) and a miniature photocell from Radio Shack were glued to opposite sides of a clip-on earring. The connectors from 


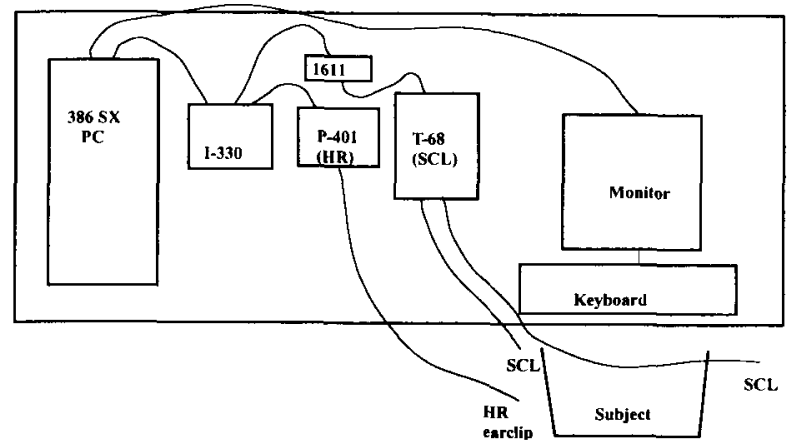

Figure 3. Equipment configuration and arrangement.

the LED and photocells were attached to the relevant wires of the J\&J photoplethysmograph pin connector (wiring diagram is available from $J \& J$ ). The ear sensor is not affected by head or hand movement and provides a stable measure of heart rate and ear pulse volume.

\section{Computer System}

Computer hardware system. We currently have the J\&J I-330 attached to a 386 SX IBM PC clone with an 80-MB hard drive. Because the biofeedback equipment is connected to the serial port of the computer, it can also be attached to a laptop or notebook, allowing for a completely portable CARMEN system. Note that a large hard drive is desirable, given that a person who types 60 words per minute will produce an output file of $100 \mathrm{~KB}$ in about 20-30 min. A large hard drive is also preferable to allow for initial data cleaning with a standard word processor.

We currently have the computer attached to a Magnavox Professional EGA Monitor-80 color monitor. The monitor can be switched from standard to monochrome text mode by a switch. This feature allows us to monitor the physiological systems in color mode and then remove them from view while the subjects type in text mode. As depicted in Figure 1, the system is situated on a table in front of the subject.

Computer software. The autonomic data from the J\&J system is converted from analog to digital by way of the I-330 interface at a sampling rate exceeding 2,400 bits per second. Heart rate and pulse volume are averaged over approximately $4 \mathrm{sec}$ to provide a relatively stable readout. The collection of the autonomic data can be accomplished by J\&J's Protool program, which is available at no charge from the J\&J company on their programmer's disk.

Our interface program is written in QBASIC. Basically, whenever the subject presses the spacebar on the keyboard, his/her autonomic levels are averaged and stored with the word preceding the space. Single SCL values from left and right hands are collected directly (in micromhos), whereas the readings from the HR system reflect the average over the previous $4 \mathrm{sec}$.

In addition to two SCL readings (left and right hands) and HR measures (heart rate and pulse volume), the subject's ID, writing condition, word number in the condition, word number in the sentence, time it took to type the word (excluding the time between words), and the actual word are saved. All of this information is saved in a data file in ASCII format. In addition, a separate file is created that includes only the text for later text analysis (see Table 1).

As the program is currently written, whenever subjects make a typing error, they can press the backspace arrow and retype. On their monitor, this appears as a boldface asterisk. We have found that this feedback ultimately discourages most subjects from trying to correct their errors. It also allows us to evaluate the autonomic (and temporal) effects of corrected versus uncorrected errors.

Table 1

Sample Output Linking Typed Text With Autonomic and Other Measures

\begin{tabular}{lccccccccc}
\hline \multicolumn{1}{c}{ Word } & ID & Con & $\begin{array}{c}\text { Tot } \\
\text { Word } \\
\text { Count }\end{array}$ & $\begin{array}{c}\text { Word } \\
\text { wi/Sent } \\
\text { Count }\end{array}$ & $\begin{array}{c}\text { Left- } \\
\text { Hand } \\
\text { SCL }\end{array}$ & $\begin{array}{c}\text { Right- } \\
\text { Hand } \\
\text { SCL }\end{array}$ & HR & PV & Time \\
\hline I & 4312d & 3 & 214 & 1 & 16.3422 & 16.6257 & 78.5106 & 18.144 & 0.02 \\
remember & $4312 \mathrm{~d}$ & 3 & 215 & 2 & 16.1784 & 16.5438 & 76.6332 & 23.499 & 1.38 \\
my & $4312 \mathrm{~d}$ & 3 & 216 & 3 & 16.1343 & 16.5438 & 76.9356 & 27.9405 & 0.22 \\
dad & $4312 \mathrm{~d}$ & 3 & 217 & 4 & 16.1847 & 16.443 & 74.2518 & 24.696 & 0.66 \\
pinning & $4312 \mathrm{~d}$ & 3 & 218 & 5 & 16.2162 & 16.3485 & 76.5324 & 22.5918 & 1.21 \\
my & $4312 \mathrm{~d}$ & 3 & 219 & 6 & 16.2414 & 16.3107 & 77.4018 & 25.8867 & 0.22 \\
mom & $4312 \mathrm{~d}$ & 3 & 220 & 7 & 16.3359 & 16.3107 & 75.2598 & 28.0539 & 0.38 \\
up & $4312 \mathrm{~d}$ & 3 & 221 & 8 & 16.3737 & 16.38 & 75.2598 & 28.0476 & 0.16 \\
against & $4312 \mathrm{~d}$ & 3 & 222 & 9 & 16.2099 & 16.317 & 77.301 & 21.2373 & 1.16 \\
the & $4312 \mathrm{~d}$ & 3 & 223 & 10 & 16.065 & 16.2855 & 78.3468 & 27.0963 & 0.17 \\
wall & $4312 \mathrm{~d}$ & 3 & 224 & 11 & 15.7815 & 16.2162 & 78.3468 & 29.5218 & 0.82 \\
and & $4312 \mathrm{~d}$ & 3 & 225 & 12 & 16.0524 & 16.1973 & 77.112 & 25.3323 & 0.28 \\
hitting & $4312 \mathrm{~d}$ & 3 & 226 & 13 & 15.9831 & 16.1784 & 76.6458 & 27.7326 & 0.88 \\
her. & $4312 \mathrm{~d}$ & 3 & 227 & 14 & 15.9516 & 16.1469 & 76.9482 & 34.7823 & 0.27 \\
\hline
\end{tabular}

Note-SCL (skin conductance level) is in micromhos (microsiemens) and HR (heart rate) in beats per minute. Pulse volume (PV, also referred to as pulse amplitude or blood volume pulse) is in relative units scaled from 0 to 100 , with higher numbers referring to greater blood volume in the appendage. Time refers to the amount of time (in seconds) elapsed from typing the first letter to the last letter of the word. ID $==$ subject's ID; Con = writing condition; Tot Word Count $=$ overall word count within the essay. Word $w i /$ Sent Count $=$ word order within the sentence. 


\section{Movement Artifacts}

By definition, we monitor autonomic activity while individuals are typing. Our initial concern was to learn if finger movements associated with typing obscured the SCL and HR measures. Observing the analog skin conductance signal while subjects type indicates that keystrokes only slightly influence SCL (typically less than 0.05 micromhos). Because most words require typing with both hands, this minor second-to-second fluctuation is divided equally across the two SCL channels. To control for this small movement, we average left- and righthand SCL for each word.

In addition to examining the SCL signals as a function of keystroke, we have also conducted an experiment in which subjects' left and right hands and left and right feet are continuously monitored (Hughes, Uhlmann, \& Pennebaker, in press). In the study of 11 college students, the mean within-subject correlation between hands and feet was approximately +.60 , ranging from .3 to .9 .

Heart rate movement artifacts are theoretically different from SCL. By definition, cardiovascular measures such as heart rate and pulse volume are affected by somatic activity (e.g., Fowles, 1980; Obrist, 1981). It would be predicted, then, that the faster an individual types, the more his/her heart rate should increase. Because we measure typing speed for each word, speed of movement can be statistically controlled. Note also that heart rate is based on a rolling average of heart interbeat intervals, and so subtle changes in typing speed from letter to letter are irrelevant for most analyses.

\section{General Data Analytic Strategy}

The data analyses for this paradigm can be quite complicated. Each subject's data are analyzed separately. Summary statistics from these individual analyses are then compiled into a separate file to examine between-subjects and between-groups effects. The general analytic strategy for each subject is as follows:

1. Cleaning the data. Using a standard word processor, each line of the subject's data is examined to adjust for run-on words, illegal characters, failure to use standard punctuation, and so on. Some people, for example, use semicolons or dashes in place of periods. These problems must be corrected by hand. Once the data are cleaned, they are converted into a standard ASCII text file and uploaded to the university's mainframe computer.

2. Statistical control of drift. SCL and, to a lesser extent, cardiovascular measures, have a tendency to drift either up or down over the course of the experiment. For SCL, a drift toward higher numbers may reflect hydration of the skin. A drift downward can also occur if one or both of the electrodes gradually loosens over the course of the experiment. Both SCL and cardiovascular measures can also drift, due to gradual relaxation or the adjustment to the laboratory room temperature.
To control for these problems, multiple regressions on the various raw $S C L$ and $H R$ measures are performed by using the word count variable (which ranges from 1 [for the first word of the essay] to whatever is the number of the last word of the essay) as the predictor. Once this regression is computed, the residual physiological measures are used to adjust each of the scores. These adjusted autonomic scores, then, serve as the basis for all subsequent analyses.

An additional concern is that different autonomic responses may lag a psychological event at different rates. Finger temperature or pulse amplitude, for example, are slower responses than changes in muscle tension (EMG). When examining text and physiological response at the phrase rather than the word level, this becomes a secondary concern. Nevertheless, for the interested researcher, it is possible to test the various lags across measures with most standard statistical packages.

3. Text analyses. Three independent judges rate each word of the essay along several predetermined categories. Phrases, rather than single words, serve as the basic unit of analysis. A phrase is defined as an independent or dependent clause with a noun and verb. Examples of text dimensions that we are assessing include positive and negative emotions, use of self-references (I or me), employment of specific defense mechanisms, active versus passive verbs, use of past, present, or future tense, and so forth. Once reliability of the judges has been assessed, their mean ratings are entered adjacent to the corrected autonomic data.

4. Linking text with autonomic activity. As the final step in analyzing the corrected raw data, multiple regressions are computed on each subject's data, using average hand SCL as the dependent measure and the various rater-based dimensions of text as the predictors. Note that for each subject, there are on average over 350 lines of data for each essay. The resultant beta weights can then be used as data within their own rights. These beta weights for each text dimension are then correlated with condition, personality measures, self-reports, and so on.

5. Other liberties we have been known to take. The data for each subject are remarkably rich in that they allow the researcher or clinician to examine subtle changes on a topic-by-topic basis. To examine specific individuals, we often plot the corrected autonomic levels with the words. In this way, we are able to see what topics and words are associated with specific physiological changes. This is of considerable clinical interest and may be quite valuable in the future. To do this, by the way, SPSSx has a number of convenient programs within their graphics and plot routines.

\section{Final Comments}

Our program and approach are still being developed. Our most recent advance, for example, allows the computer to ask the subject a series of open-ended questions 
or even survey true-false questions. Autonomic levels are stored for each word typed on the screen by either the computer program itself or the subject.

It should be noted that it is theoretically possible to develop an inexpensive alternative to the $J \& J$ system, especially for the continuous measurement of skin conductance and heart rate.

\section{Availability}

We can supply researchers with the basic computer program to run this project. In return, we ask you to share with us any significant advances in your own programming approach. Researchers who would like a free copy of the computer program described in this paper should send a blank formatted DOS (3.5 or 5.25 in., any density) to the author.

\section{REFERENCES}

EDELBERG, R. (1967). Electrical properties of the skin. In C. C. Brown (Ed.), Methods in psychophysiology (pp. 1-53). Baltimore: Williams \& Wilkins.

FowLEs, D. C. (1980). The three arousal model: Implications of Gray's two-factor theory for heart rate, electrodermal activity, and psychopathy. Psychophysiology, 17, 87-104.

Hughes, C. F., Uhlmann, C., \& Pennebaker, J. W. (in press). The body's response to psychological defenses: Linking verbal text with autonomic activity. Journal of Personality.

OвRIST, P. A. (1981). Cardiovascular psychophysiology: A perspective. New York: Plenum.

Pennebaker, J. W., Hughes, C. F., \& O'Heeron, R. C. (1987). The psychophysiology of confession: Linking inhibitory and psychosomatic processes. Journal of Personality \& Social Psychology, 52, 781-793.

(Manuscript received May 21, 1992; revision accepted for publication January $25,1993$. 